\title{
Mothers' psychological adaptation to Duchenne/Becker muscular dystrophy
}

\author{
Holly L Peay ${ }^{\star, 1,2}$, Bettina Meiser ${ }^{3}$, Kathleen Kinnett ${ }^{1}$, Pat Furlong ${ }^{1}$, Kathryn Porter $^{1}$ and Aad Tibben ${ }^{2}$ \\ Duchenne and Becker muscular dystrophy (DBMD) cause significant emotional and care-related burden on caregivers, but no \\ studies have evaluated predictors of positive caregiver outcomes, including disorder-specific psychological adaptation. Using a \\ community-engaged approach focused on supporting mothers in positive aspects of caregiving, this prospective study aims to \\ assess (i) the association between child's baseline functional status and mothers' illness perceptions, resilience, and coping self- \\ efficacy; and (ii) predictors of mothers' psychological adaptation to caring for a child with DBMD. Biological mothers with at \\ least one living child with DBMD completed a baseline survey $(n=205)$ with 1 -year $(n=147)$ and 2 -year $(n=144)$ follow-up \\ surveys. Worse child's baseline function was associated not only with increased caregiver burden and reduced maternal \\ resilience, but also with perception of positive disease impact on the family. At two follow-ups, increased psychological \\ adaptation to DBMD was predicted by resilience $(\beta=0.264, P=0.001)$ and perceived positive impact $(\beta=0.310, P<0.001)$, \\ controlling for mother's age $(\beta=-0.305, P<0.001)$ and income $(\beta=-0.088, P=0.245)$. Child's functional status and \\ caregiver burden of DBMD did not predict DBMD-specific adaptation. Though clinicians caring for families with DBMD should \\ anticipate increased caregiver burden as the disorder progresses, interventions focused on caregiver burden are not expected to \\ influence mothers' psychosocial adaptation. Efforts to improve mothers' well-being should focus on fostering mothers' resilience \\ and enhancing perceptions of positive disease impact (benefit finding). Results suggest that psychosocial interventions can \\ highlight strengths and well-being rather than burden and deficit.
}

European Journal of Human Genetics (2016) 24, 633-637; doi:10.1038/ejhg.2015.189; published online 26 August 2015

\section{INTRODUCTION}

Duchenne and Becker muscular dystrophy (DBMD) are rare, progressive, $\mathrm{X}$-linked diseases of muscle wasting caused by mutations that alter production of the dystrophin protein. ${ }^{1}$ Duchenne muscular dystrophy is the more common and severe of the two disorders, with noticeable symptoms in early pediatric years. ${ }^{2}$ It leads to severe progressive muscle weakness that results in increasing care needs as the child ages, and death typically in the late $20 s^{3-4}$ Becker muscular dystrophy is more heterogeneous, ranging from a course similar to Duchenne to milder presentations with later onset and more slowly progressing weakness. ${ }^{2}$

Several cross-sectional studies have explored the impact of caring for a child with DBMD on parent/guardian caregivers. Studies have shown high caregiving demands, ${ }^{5-7}$ stress, ${ }^{6,8,9}$ distress, ${ }^{6,10}$ and lower health-related quality of life in caregivers. ${ }^{11}$ Psychological outcomes have been found to be associated with the child's illness progression, perceived caregiving burden, social support, and financial burden. $5,6,9,10$ However, survey and interview studies have also described components of DBMD caregiving that were perceived as beneficial, including an improved ability to appreciate life experiences and other positive coping strategies to manage the progressive disease course and chronic sorrow. ${ }^{12-15}$ Pangalila and colleagues' ${ }^{5}$ cross-sectional study of parents of adults with DBMD found not only high subjective burden, but also high rating of care as important and rewarding. Kenneson and Bobo ${ }^{6}$ found that general life satisfaction was associated with high social support, high resiliency, and high income.
To date, no studies have evaluated disorder-specific adaptation in caregivers of individuals with BDMD. Disorder-specific adaptation is defined as a process of coming to terms with the implications of a health threat and the outcomes of that process. ${ }^{16}$ Across patients and caregivers, factors reported to be associated with adaptation include personality traits such as optimism; economic resources; social support; cognitive and emotional appraisals of the condition, including disease impact and control; and coping strategies and efforts to make meaning of the disease experience. ${ }^{16-18}$

Our choice of psychological adaptation to DBMD as the study outcome is consistent with the preferences expressed during community engagement (described below). Predictor variables were chosen based on the adaptation literature, studies of DBMD caregivers, and through community engagement. The illness perceptions in this study included worry about the child's care and mothers' perceptions of control, caregiver burden, and positive impact of DBMD. Personal attributes included dispositional optimism, a personality trait associated with psychological well-being and physical health across a range of disease populations; ${ }^{19}$ and resilience, which measures 'protective resources' that have been demonstrated to facilitate flexibility in coping. ${ }^{20}$ We also measured coping self-efficacy, which assesses perceived ability to cope with challenges and threats. ${ }^{21}$ On the basis of the importance of disease progression on caregiver outcomes in the DBMD literature, ${ }^{6,11}$ the functional status of the child with DBMD was also assessed. This longitudinal study includes data from three survey points (baseline, year 1, and year 2) and aims to: (i) assess the

\footnotetext{
${ }^{1}$ Parent Project Muscular Dystrophy, Hackensack, NJ, USA; ${ }^{2}$ Department of Clinical Genetics, Leiden University Medical Centre, Leiden, The Netherlands; ${ }^{3}$ Prince of Wales Clinical School, University of New South Wales, Sydney, New South Wales, Australia

${ }^{*}$ Correspondence: Dr HL Peay, Parent Project Muscular Dystrophy, 401 Hackensack Avenue, 9th Floor, Hackensack, NJ 07601 , USA. Tel: +1 443791 5927; Fax: +1 201 250 8435 ; E-mail: holly@parentprojectmd.org

Received 21 October 2014; revised 11 June 2015; accepted 12 July 2015; published online 26 August 2015
} 
associations between child's functional status and mothers' resilience, illness perceptions and coping self-efficacy; and (ii) determine whether child's functional status and mothers' personal attributes, illness perceptions, and coping self-efficacy as measured at baseline predict mothers' psychological adaptation to DBMD 2 years later.

It was hypothesized that the child's functioning at the time of the baseline survey would be associated with mothers' resilience, illness perceptions, and coping self-efficacy-specifically, that worse child function (ie, more severe illness) would be associated with negative impacts on resilience, illness perceptions, and coping self-efficacy. It was also hypothesized that mothers' psychological adaptation measured 2 years later would be predicted by better child function and higher levels of dispositional optimism, resilience, and coping selfefficacy.

Data on predictors of adaptation are needed to inform the development of interventions aimed at improving caregivers' wellbeing. By pinpointing when interventions may be most necessary and identifying which caregivers are most likely to develop adverse psychological outcomes, interventions can be targeted more precisely. Longitudinal data collection is advantageous because, in contrast to cross-sectional designs, it allows assessment of causality in assessing predictors of psychological adaptation.

\section{MATERIALS AND METHODS}

\section{Methodological approach}

This study used a community-engaged research approach, in which advocates, clinicians, caregivers, and social science researchers (identified as 'the community') identified the research agenda, design, and delivery. ${ }^{22,23}$ Most notably, a community-engaged approach impacted the research agenda; that is, the focus of the project was to be responsive to a community-identified need to appreciate and explore positive outcomes experienced by caregivers, and then develop interventions focused on improving adaptation rather than solely target negative impact on caregivers.

This longitudinal survey study was determined to be exempt by the Cincinnati Children's Hospital Medical Center Institutional Review Board. This study was undertaken as part of a larger study that also assessed unmet needs, the results of which are reported separately.

\section{Participants}

Participants were biological mothers of at least one living child with Duchenne or Becker muscular dystrophy, who lived in the United States, were 18 years of age or older, and able to answer questionnaires in English. The online questionnaire was implemented using SurveyMonkey software. Participants completed the baseline survey between November 2011 and October 2012, followed by two follow-up surveys, which were distributed 1 year and 2 years later.

\section{Recruitment strategy}

Recruitment was conducted through online and e-mailed advertisements and social media postings through the Duchenne Connect Registry (www.duchenneconnect.org) and Parent Project Muscular Dystrophy, and continued through snowball recruitment; and through face-to-face invitations and advertisements distributed at Cincinnati Children's Hospital Medical Center neuromuscular clinic. The total number of participants invited to the study is unknown given the variety of recruitment approaches, the use of social media and snowball recruiting, and overlap among the recruitment populations.

\section{Procedure}

Eligible mothers provided their contact information and each participant was asked to complete a baseline questionnaire. With the exception of two participants who requested mailed paper copies, participants responded to surveys online using unique survey links for each participant. The unique links to follow-up surveys were sent by e-mail at about 12 and 24 months after the baseline survey.

\section{Measures}

Demographic/disease characteristics. Participants' age, ethnicity, education, marital status, income, employment status, state of residence, and mother's carrier status were assessed. Additional items related to the child included: diagnosis (Duchenne, Becker, or intermediary phenotype), current age, age at diagnosis, and functional status. The latter was measured using a 7-item categorization, where a higher score means worse condition. The same functional assessment item has been used in the DuchenneConnect patient registry and is an adaptation of the stages in the Duchenne care guidelines. ${ }^{4}$

Personal attributes. Dispositional optimism was measured with the 10-item Life Orientation Test Revised (LOT-R). ${ }^{19}$ Cronbach's alpha in this sample was 0.87 . Resilience was measured using the Resilience Scale for Adults (RSA). ${ }^{20}$ Cronbach's alpha was 0.94 .

Illness perceptions. Perceived Caregiver Burden was measured using the 12 -item Zarit Burden Interview (ZBI). ${ }^{24} \mathrm{~A}$ score of 17 or above may be used as the cutoff point to identify high burden. ${ }^{24}$ Cronbach's alpha was 0.89 . Perceived Personal Control was measured using five questions about control over DBMD in general, daily symptoms, longterm course, medical care and treatment, and control by others (adapted from Lipinski and colleagues). ${ }^{25}$ Cronbach's alpha was 0.79 . Worry about Care for Child with DBMD was measured using three items purposively designed to assess amount, frequency, and intensity of DBMD-specific care worry (developed as suggested by McCaul and Goetz). ${ }^{26}$ Cronbach's alpha was 0.89. Perceived Positive Impact was measured with one item purposively developed for the study ('How much of a positive effect does your child's condition have on your entire family?').

Coping Self-Efficacy. Coping Self-Efficacy was assessed with the Coping Self-Efficacy Scale. ${ }^{21}$ Cronbach's alpha was 0.97.

Psychological Adaptation. Psychological Adaptation wsas measured with the 20-item Psychological Adaptation Scale (PAS), which is designed to measure adaptation to a chronic condition or disease risk by patients or caregivers. ${ }^{17}$ Cronbach's alpha was 0.96 .

\section{Statistical analyses}

Data were initially explored with descriptive statistics. Measures of internal consistency (Cronbach's alpha) were conducted using baseline survey data. The scale/item means for resilience, perceived caregiver burden, perceived control, coping self-efficacy, and psychological adaptation were assessed for changes across the three survey time points (baseline, year 1, and year 2) using repeated-measures ANOVA, and the median child's functional status using a Friedman test.

Spearman correlations were used to assess bivariate associations of baseline child's functional status with mothers' resilience, perceived caregiver burden, perceived control, worry about care, perceived positive impact, and coping self-efficacy. Bivariate relationships among variables measured at baseline (child's functional status, dispositional optimism, worry about care, perceived control, caregiver burden, resilience, coping self-efficacy) and mothers' psychological 
adaptation to DBMD measured at the 1-year and 2-year follow-up surveys were then examined using Pearson or Spearman correlations, as appropriate.

To assess the predictors of psychological adaptation 2 years later, all predictor variables with $P<0.25$ in the bivariate analysis were entered into a multiple linear regression, then progressively eliminated until only those with $P$-values of $<0.05$ remained. Potential confounders (mother's age, income, carrier status) were then entered one at a time and retained in the regression if the $\beta$ associated with any of the predictor variables changed by more than $10 \%$.

A post hoc correlation analysis was conducted to assess associations between mothers' age and child's functional status, resilience, and perceived positive impact. This was followed by a partial correlation to explore the relationship between mothers' age and psychological adaptation, while controlling for child's functional status.

For all analyses, total measure scores rather than sub-scores were utilized. Child's functional status was chosen as a predictor rather than child's age or diagnosis because the clinical variability in the DBMD diagnostic categories ${ }^{2,4}$ makes anticipation of natural history or stage based on age difficult. Further, given the lower prevalence of Becker muscular dystrophy, it was anticipated that the number of mothers with sons with the latter type of dystrophy would be too small to allow statistically meaningful comparisons with Duchenne muscular dystrophy.

\section{RESULTS}

Sample

Two hundred and five mothers participated in the baseline survey, 147 participated in the follow-up survey 1 year later, and 144 participated in the follow-up survey 2 years later (a $30 \%$ loss rate from the baseline survey cohort). This includes two mothers who completed the baseline survey but did not complete the 2-year follow-up because their affected child died between survey points.

At the time of the baseline survey, 192 (93.2\%) were identified as Caucasian, $11(5.3 \%)$ as Hispanic, $6(2.9 \%)$ as Asian, and $7(3.4 \%)$ as 'other'; respondents had the option of endorsing more than one category. The mean age of the mothers was 44.0 years $(\mathrm{SD}=8.7)$, with a range of 27-71. The majority of participants had at least a college degree $(136,67.4 \%)$ and was employed or attending school part- or full-time $(145,71.5 \%)$. The median household income was $\$ 50000$ $\$ 99$ 999. One hundred and seventy-seven $(86.3 \%)$ were married or in a long-term committed relationship, $24(11.7 \%)$ were divorced or separated, $3(1.5 \%)$ had never married, and $1(0.5 \%)$ was widowed. Ninety-six participants $(46.8 \%)$ reported being DBMD carriers, 78 $(38.0 \%)$ were non- carriers, and $31(15.1 \%)$ did not know whether they were carriers.
The majority of participants had one affected child (184, 89.8\%), 19 (9.3\%) had two affected children, and $2(1.0 \%)$ had three affected children. One hundred and seventy-four $(84.9 \%)$ of the affected children had DMD, 23 (11.2\%) had BMD, and 8 (3.9\%) had an intermediate phenotype. The mean age of the affected child was 13.8 years $(\mathrm{SD}=7.2)$, with a range of $1-40$ years. Mean child functional status was $3.5(\mathrm{SD}=1.8, N=205)$ at baseline and $3.9(\mathrm{SD}=1.8$, $N=144$ ) at 2 -year follow-up, with higher values indicating worse function. If the participant had more than one affected child, the functional status of the oldest living child is reported.

There were no statistically significant differences in mean age for those who answered the survey at baseline and time two $(M=46.12$, $\mathrm{SD}$ 8.77) and those who answered the baseline only $(M=43.19$, $\mathrm{SD}=9.04, P=0.10)$; or a difference in income for those who answered the survey at baseline and time two $(\mathrm{M}=2.65, \mathrm{SD}=1.36)$ versus those who answered at baseline only $(\mathrm{M}=2.35, \mathrm{SD}=1.22, P=0.23)$. Mean child functional status demonstrated a small but significant change from those who answered both the baseline survey and the survey at time two $(\mathrm{M}=3.15, \mathrm{SD}=1.86)$ and those who answered at baseline only $(\mathrm{M}=3.91, \mathrm{SD}=1.72, P=0.03)$.

Table 1 presents means and standard deviations of mothers' perceptions of BDMD caregiving, personal attributes, coping selfefficacy, and adaptation at baseline, 1 year and 2 years later; and mean and median for child's functional status. Defining a high ZBI score as 17 or above, ${ }^{24} 48.2 \%$ of the mothers reported high caregiver burden at baseline, $55.1 \%$ at 1 -year follow-up, and $52.8 \%$ of the mothers reported high burden at the two-year follow-up.

Table 2 Pearson and Spearman correlations among child function and predictors at baseline and psychological adaptation (PAS) at 1 -year and 2-year follow-up

\begin{tabular}{|c|c|c|c|}
\hline Scale/item at baseline & Child function & $\begin{array}{c}\text { PAS } \\
\text { (1-year follow-up) }\end{array}$ & $\begin{array}{c}\text { PAS } \\
\text { (2-year follow-up) }\end{array}$ \\
\hline Child function & - & 0.089 & 0.008 \\
\hline Optimism (LOT-R) & 0.064 & $0.199^{a}$ & $0.190^{\mathrm{a}}$ \\
\hline Resilience (RSA) & $-0.141^{a}$ & $0.343^{b}$ & $0.330^{\mathrm{b}}$ \\
\hline Caregiver burden (ZBI) & $0.323^{b}$ & -0.155 & -0.117 \\
\hline Perceived control & 0.045 & $0.302^{b}$ & $0.229^{a}$ \\
\hline Worry about child's care & 0.100 & -0.148 & -0.084 \\
\hline Perceived positive impact & $0.145^{\mathrm{a}}$ & $0.376^{\mathrm{b}}$ & $0.399^{b}$ \\
\hline Coping self-efficacy & 0.042 & $0.362^{\mathrm{b}}$ & $0.348^{b}$ \\
\hline
\end{tabular}

${ }^{a}$ Correlation is significant at the 0.05 level (two-tailed). bCorrelation is significant at the 0.01 level (two-tailed).

Table 1 Measure/item means: baseline 1-year and 2-year follow-up

\begin{tabular}{|c|c|c|c|c|}
\hline & Scale/item range & Baseline mean $(n=205)$ & 1-year mean $(n=147)$ & 2-year mean $(n=144)$ \\
\hline Child function ${ }^{a}$ & $1-7$ & $3.5(\mathrm{SD}=1.8)$ Median $=3.0$ & $3.7(\mathrm{SD}=1.7)$ Median = 3.0 & $3.9(\mathrm{SD}=1.8)$ Median $=4.0$ \\
\hline Optimism (LOT-R) & $0-24$ & $14.3(\mathrm{SD}=4.6)$ & - & - \\
\hline Resilience (RSA) & $1-5$ & $3.9(\mathrm{SD}=0.5)$ & $3.9(\mathrm{SD}=0.6)$ & $3.9(\mathrm{SD}=0.5)$ \\
\hline Caregiver burden (ZBI) & $0-48$ & $17.1(\mathrm{SD}=8.6)$ & $17.6(\mathrm{SD}=8.5)$ & $17.7(\mathrm{SD}=8.6)$ \\
\hline Perceived control ${ }^{\mathrm{b}}$ & $1-11$ & $5.5(\mathrm{SD}=2.1)$ & $5.4(\mathrm{SD}=2.0)$ & $4.6(\mathrm{SD}=2.1)$ \\
\hline Worry about child's care & $3-15$ & $7.3(\mathrm{SD}=2.9)$ & $7.1(\mathrm{SD}=2.7)$ & - \\
\hline Perceived positive impact & $0-10$ & $5.6(\mathrm{SD}=2.9)$ & $5.7(\mathrm{SD}=2.8)$ & - \\
\hline Coping self-efficacy ${ }^{b}$ & $0-260$ & $156.5(\mathrm{SD}=51.6)$ & $162.8(\mathrm{SD}=47.6)$ & $168.4(\mathrm{SD}=49.4)$ \\
\hline Psychological adaptation (PAS) & $1-5$ & $3.5(\mathrm{SD}=0.9)$ & $3.6(\mathrm{SD}=1.0)$ & $3.6(\mathrm{SD}=1.0)$ \\
\hline
\end{tabular}

aChange in median significant at the $P=0.005$ level.

${ }^{b}$ Change in mean significant at the $P=0.01$ level (2-tailed). 
Repeated measures ANOVA demonstrated significant reduction in perceived control (Wilks' Lambda $=0.83, \mathrm{~F}(2114)=11.61$, $P<0.005$ ) and a significant increase in coping self-efficacy (Wilks' Lambda $=0.90, \mathrm{~F}(2114) 6.26, P=0.003$ ) across the three survey time points. A Friedman test revealed a significant difference in median child's functional status $\left(\chi^{2}(2, n=118)=55.68, P<0.005\right)$, demonstrating the expected reduction in child's function over the 2 years of data collection.

\section{Bivariate analyses}

Worse child's functional status was significantly associated with lower resilience, higher perceived caregiver burden, and higher perceived positive impact at the time of the baseline survey (Table 2). Analysis of predictor variables (as measured at baseline) and psychological adaptation (as measured at 2-year follow-up) showed statistically significant, positive associations between the predictor variables dispositional optimism, resilience, perceived control, positive impact, and coping self-efficacy, and the outcome variable psychological adaptation.

\section{Multivariate analyses of psychological adaptation}

A multiple linear regression was performed to assess predictors of psychological adaptation at the 2-year follow-up. The final model (see Table 3) included perceived positive impact $(\beta=0.310, P<0.001)$ and resilience $(\beta=0.264, \quad P=0.001)$, which explained $30.5 \%$ of the variance in mothers' psychological adaptation to DBMD, after controlling for effects of participants' age $(\beta=-0.305, P<0.001)$ and income $(\beta=-0.088, P=0.245)$. Child's functional status, worry about child's care, perceived control, perceived caregiver burden, dispositional optimisms, and coping self-efficacy did not predict psychological adjustment.

On post hoc analyses, mother's age was positively correlated with child's function (Spearman correlation $=0.486, P<0.001$ ). However, age was not associated with resilience $(\mathrm{r}=-0.002, P=0.978)$ or perceived positive impact $(r=-0.033, P=0.650)$. There was a negative partial correlation between mothers' age and psychological adaptation at the 2-year follow-up even while controlling for child's functional status $(\mathrm{r}=-0.389, n=135, P<0.0005)$ with younger mothers' age being associated with higher levels of psychological adaptation. An inspection of the zero-order correlation $(r=-0.336)$ suggests that controlling for functional status had very little effect on the strength of the relationship between those two variables.

\section{DISCUSSION}

Consistent with previously reported studies, ${ }^{5-7}$ this study found that worse functional status in the child was associated with higher perceived caregiver burden, and approximately 50\% of mothers reported a high level of burden. Thus, families, clinicians, and other stakeholders might anticipate increased caregiving burden as the

Table 3 Predictors of psychological adaptation at 2-year follow-up $(N=136)$

\begin{tabular}{lcrr}
\hline Variable & Beta coefficient & $T$ & P value \\
\hline Perceived positive impact & 0.310 & 4.122 & $<0.001$ \\
Resilience & 0.264 & 3.459 & 0.001 \\
Mothers' age & -0.305 & -4.163 & $<0.001$ \\
Household income & -0.088 & -1.169 & 0.245 \\
\hline
\end{tabular}

Final model: $\mathrm{R}^{2}=0.325, F(4,136)=15.889, P<0.001$.

Adjusted $\mathrm{R}^{2}=0.305, \mathrm{R}=0.570$. disorder progresses. Worse child functional status was associated with lower mothers' resilience but higher perceived positive impact, suggesting that although mothers are able to find benefits associated with the DBMD experience as symptoms progress, their resilience may be challenged.

However, the longitudinal results provide important additional insights: that neither perceived caregiver burden nor child's functional status predicted psychological adaptation 2 years later. Instead, better adaptation was predicted by increased resilience and perceived positive impact. Resilience is a multidimensional personal attribute that may be shaped by personality traits, evolving appraisals, social support systems, and family environments. ${ }^{20,27-29}$ Simply defined, it is a relative resistance to adversity $^{30}$ that has been demonstrated to influence positive outcomes after a stressful event. ${ }^{29,31-32}$ As measured by the Resilience Scale for Adults, it comprises protective personal and social resources. ${ }^{20}$ Consistent with our study's community-engaged focus on evaluating predictors of positive, DMD-focused psychological outcomes, the emerging field of positive psychology highlights resilience as a personal strength that fosters not only recovery from an adverse event, but also as a contributor to personal growth. ${ }^{33}$

The concept of personal growth is also represented by mothers' endorsement of positive impact. This endorsement reflects benefit finding - a perception that major positive changes can come from a traumatic life experience. ${ }^{34}$ Across a range of conditions, the ability to find benefit in a health threat has been associated with improved psychosocial and health outcomes. ${ }^{35-38}$

Contrary to our hypothesis, there was no association between a child's functional status and psychological adaptation, and yet mothers' age confounded the relationships of positive predictive impact and resilience with psychological adaptation. Post hoc analysis showed that there is a negative association between age and psychological adaptation that is not accounted for by child's functional status. In addition, mothers' age was not significantly associated with resilience or perceived positive impact, the predictors of adaptation. It is possible that other predictors may vary by age and influence psychological adaptation, such as mothers' hope for a better outcome for their child, their spirituality and religious beliefs, and their capacity to manage the physical demands of caretaking as the disorder progresses. Additional exploration of these and other variables is possible in this longitudinal cohort, and is needed to better understand the influence of increasing mother's age and worsening child's functional status on the predictors of long-term, disease-specific psychological adaptation.

Before describing clinical implications of the results, the study's strengths and limitations should be discussed. The study had a prospective design, allowing causal associations to be demonstrated between predictors and psychological adaptation. Though 30\% of participants were lost to follow-up, there was no evidence of participation bias in terms of mothers' age or income level. There was a small but significant worsening in child's functional status for those who completed the survey at baseline only versus those who answered at baseline and time two, possibly related to increased caregiver burden leading to less time for participation. This may have implications for the interpretation of the longitudinal results.

The study sample was broadly recruited through diverse sources, thus increasing the representativeness of the sample. However, the study is limited by the opt-in nature of the recruitment process, which may have generated selection bias. This could not be evaluated as details on individuals who did not participate were not available. In addition, the response rate cannot be evaluated given the variety of recruitment approaches, the use of social media and snowball 
recruiting, and overlap among the recruitment populations. Traditionally underserved minority and low SES populations were underrepresented in the survey, as were non-married mothers, which may have implications for the generalizability of the findings. The study should be repeated in an entirely clinic-based sample to allow ascertainment of a more representative sample and to increase generalizability of the data.

\section{Clinical implications}

Efforts to improve mothers' well-being should focus on fostering mothers' resilience and enhancing benefit finding through identification of positive aspects of living with DBDM on the caregiver and family. Interventions that aim to increase resilience should be evaluated in both clinical and disease advocacy settings. Though additional research is needed to translate findings in the resilience literature into the development of effective interventions, ${ }^{29}$ several interventions have been proposed to improve resilience. ${ }^{39-40}$ In addition, further research should evaluate the potential benefit of routine assessment of perceived positive impact, possibly through using the single item used in this study. Such assessment may identify mothers to whom psychosocial interventions may be targeted. In contrast, our findings suggest that interventions targeted at caregiver burden might not be an effective method to improve mothers' long-term psychological well-being. Overall, the results of this study suggest that clinical and psychosocial interventions can highlight strengths and well-being rather than burden and deficit.

\section{CONFLICT OF INTEREST}

The authors declare no conflict of interest.

\section{ACKNOWLEDGEMENTS}

We are grateful to the women who participated in this study. Sheila Moeschen provided valuable input into the survey development. Hadar Scharff provided database coordination and administrative support. Associate Professor Bettina Meiser received a Career Development Award Level 2 and a Senior Research Fellowship Level B from the National Health and Medical Research Council of Australia.

1 Hoffmann EP, Brown RH, Kunkel LM: Dystrophin: the protein product of the Duchenne muscular dystrophy locus. Cell 1987; 51: 919-928.

2 Flanigan KM: Duchenne and Becker muscular dystrophies. Neurol Clin 2014; 32: 671-688.

3 Eagle M, Baudouin SV, Chandler C, Giddings DR, Bullock R, Bushby K: Survival in Duchenne muscular dystrophy: improvements in life expectancy since 1967 and the impact of home nocturnal ventilation. Neuromuscul Disord 2002; 12 926-929.

4 Bushby K, Finkel R, Birn'krant DJ et al: Diagnosis and management of Duchenne muscular dystrophy: part 1: diagnosis, and pharmacological and psychosocial management. Lancet Neurol 2010; 9: 77-93.

5 Pangalila RF, van den Bos GA, Stam HJ, van Exel NJ, Brouwer WB, Roebroeck ME: Subjective caregiver burden of parents of adults with Duchenne muscular dystrophy. Disabil Rehabil 2012; 34: 988-996.

6 Kenneson A, Bobo JK: The effect of caregiving on women in families with Duchenne/Becker muscular dystrophy. Health Soc Care Community 2010; 18 520-528.

7 Boyer F, Drame M, Morrone I, Novella JL: Factors relating to carer burden for families of persons with muscular dystrophy. J Rehabil Med 2006; 38: 309-315.

8 Nereo NE, Fee RJ, Hinton VJ: Parental stress in mothers of boys with duchenne muscular dystrophy. J Pediatr Psychol 2003; 28: 473-484.

9 Reid DT, Renwick RM: Relating familial stress to the psychosocial adjustment of adolescents with Duchenne muscular dystrophy. Int J Rehabil Res 2001; 24: 83-93.

10 Daoud A, Dooley JM, Gordon KE: Depression in parents of children with Duchenne muscular dystrophy. Pediatr Neurol 2004; 31: 16-19.

11 Hatzmann J, Heymans HS, Ferrer-i-Carbonell A, van Praag BM, Grootenhuis MA Hidden consequences of success in pediatrics: parental health-related quality of liferesults from the Care Project. Pediatrics 2008; 122: e1030-e1038.
12 Erby LH, Rushton C, Geller G: 'My son is still walking': stages of receptivity to discussions of advance care planning among parents of sons with Duchenne muscular dystrophy. Semin Pediatr Neurol 2006; 13: 132-140.

13 Kinnett K, Eakes GG, Cripe L: Duchenne muscular dystrophy: More adults are reaching adulthood - and facing new challenges with the disease. Advance Health Network August 8, 2010. Available from: http://nurse-practitioners-and-physicianassistants.advanceweb.com/features/articles/duchenne-muscular-dystrophy.aspx (last accessed 15 December 2014).

14 Webb CL: Parents' perspectives on coping with Duchenne muscular dystrophy. Child Care Health Dev 2005; 31: 385-396.

15 Samson A, Tomiak E, Dimillo J et al: The lived experience of hope among parents of children with Duchenne muscular dystrophy: perceiving the human beyond the illness. Chronic IIIn 2009; 5: 103-114.

16 Biesecker BB, Erby L: Adaptation to living with a genetic condition or risk: a minireview. Clin Genet 2008; 74: 401-407.

17 Biesecker BB, Erby LH, Woolford S et al: Development and validation of the Psychological Adaptation Scale (PAS): use in six studies of adaptation to a health condition or risk. Patient Educ Couns 2013; 93: 248-254.

18 Peay HL, Rosenstein DL, Biesecker BB: Adaptation to bipolar disorder and perceived risk to children: a survey of parents with bipolar disorder. BMC Psychiatry 2013; 13 . 327.

19 Scheier MF, Carver CS, Bridges MW: Distinguishing optimism from neuroticism (and trait anxiety, self-mastery, and self-esteem) - a reevaluation of the life orientation test. J Pers Soc Psychol 1994; 67: 1063-1078.

20 Friborg O, Barlaug D, Martinussen M, Rosenvinge $\mathrm{JH}$, Hjemdal O: Resilience in relation to personality and intelligence. Int J Method Psych 2005; 14: 29-42.

21 Chesney MA, Neilands TB, Chambers DB, Taylor JM, Folkman S: A validity and reliability study of the coping self-efficacy scale. $\mathrm{Br} J$ Health Psychol 2006; 11: 421-437.

22 Staley K Exploring impact: public involvement in NHS, public health and social care research. INVOLVE 2009. Available at: http://www.invo.org.uk/posttypepublication/ exploring-impact-public-involvement-in-nhs-public-health-and-social-care-research/\# (accessed 16 October 2014).

23 Clinical and Translational Science Awards Consortium Community Engagement Key Function Committee Task Force on the Principles of Community Engagement: Principles of Community Engagement, 2nd Edition. Agency for Toxic Substances and Disease Registry, National Institutes of Health publication \#11-7782. 2011. Available at: http://www.atsdr.cdc.gov/communityengagement (accessed 15 October 2014).

24 Bédard M, Molloy DW, Squire L, Dubois S, Lever JA, O'Donnell M: The Zarit Burden Interview: a new short version and screening version. Gerontologist 2001; 41: 652-657.

25 Lipinski SE, Lipinski MJ, Biesecker LG, Biesecker BB: Uncertainty and perceived personal control among parents of children with rare chromosome conditions: the role of genetic counseling. Am J Med Genet C Semin Med Genet 2006; 142C 232-240.

26 McCaul KD, Goetz PW Worry; in National Cancer Institute: Health Behavior Constructs: Theory, Measurement \& Research. Available at: http://cancercontrol.cancer.gov/brp/ constructs/worry/w6.html (accessed 16 October 2014).

27 Rosenberg AR, Baker KS, Syrjala KL, Back AL, Wolfe J: Promoting resilience among parents and caregivers of children with cancer. J Palliat Med 2013; 16 : 645-652.

28 Ahern NR, Kiehl EM, Sole ML, Byers J: A review of instruments measuring resilience. Issues Comp Pediatr Nurs 2006; 29: 103-125.

29 Rutter M: Annual research review: Resilience — clinical implications. J Child Psychol Psychiat 2013; 54: 474-487.

30 Rutter M: Resilience concepts and findings: Implications for family therapy. J Fam Ther 1999; 21: 119-144.

31 Rew L, Horner SD: Youth resilience framework for reducing health-risk behaviors in adolescents. J Pediatr Nurs 2003; 18: 379-388.

32 Masten A, Reed M: Resilience in development. In: Snyder CR, Lopez SJ (eds): Handbook of Positive Psychology. Oxford University Press: Oxford, 2005, pp 74-88.

33 Bonanno G: Loss, trauma and human resilience: Have we underestimated the human capacity to thrive after extremely aversive events? Am Psychologist 2004; 59: 20-28.

34 Lechner SC: Benefit finding In: Lopez SJ (eds): The Encyclopedia of Positive Psychology. London, UK: Blackwell Publishing, 2009. Blackwell Reference Online. Available at: http://www.blackwellreference.com/public/book.html?id=g9781405161251_97814 05161251 (accessed 16 October 2014).

35 Barskova T, Oestereich R: Post-traumatic growth in people living with a serious medical condition and its relations to physical and mental health: A systematic review. Disabil Rehabil 2009; 31: 1709-1733.

36 Bower JE, Moskowitz JT, Epel E: Is benefit finding good for your health? Pathways linking positive life changes after stress and physical health outcomes. Curr Dir Psychol Sci 2009; 18: 337-341.

37 Danoff-Burg S, Revenson TA: Benefit-finding among patients with rheumatoid arthritis: Positive effects on interpersonal relationships. J Behav Med 2005; 28: 91-103.

38 Low CA, Stanton AL, Danoff-Burg S: Expressive disclosure and benefit finding among breast cancer patients: mechanisms for positive health effects. Health Psychol 2006; 25: 181-189.

39 Connor K, Davidson J: Development of a new resilience scale: The Connor-Davidson Resilience Scale (CD-RISC). Depress Anxiety 2003; 18: 76-82.

40 Newman R: APA's resilience initiative. Profess Psychol Res Pr 2005; 36: 227-229. 